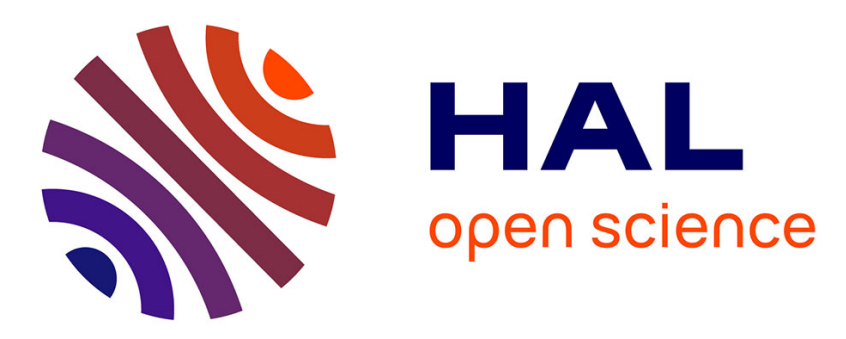

\title{
Symmetry breakings in a metal organic framework with a confined guest
}

Céline Mariette, Elzbieta Trzop, Jean-Yves Mevellec, Abdou Boucekkine, Aziz

Ghoufi, Guillaume Maurin, Eric Collet, M. Carmen Muñoz, José Antonio Real, Bertrand Toudic

\section{To cite this version:}

Céline Mariette, Elzbieta Trzop, Jean-Yves Mevellec, Abdou Boucekkine, Aziz Ghoufi, et al.. Symmetry breakings in a metal organic framework with a confined guest. Physical Review B, 2020, 101 (13), 10.1103/PhysRevB.101.134103 . hal-02552322

\section{HAL Id: hal-02552322 \\ https://hal.umontpellier.fr/hal-02552322}

Submitted on 28 May 2020

HAL is a multi-disciplinary open access archive for the deposit and dissemination of scientific research documents, whether they are published or not. The documents may come from teaching and research institutions in France or abroad, or from public or private research centers.
L'archive ouverte pluridisciplinaire HAL, est destinée au dépôt et à la diffusion de documents scientifiques de niveau recherche, publiés ou non, émanant des établissements d'enseignement et de recherche français ou étrangers, des laboratoires publics ou privés. 


\section{Symmetry breakings in a Metal Organic Framework with Confined Guest}

Céline Mariette, ${ }^{1, *}$ Elżbieta Trzop,${ }^{1}$ Jean-Yves Mevellec, ${ }^{2}$ Abdou Boucekkine, ${ }^{3}$ Aziz Ghoufi, ${ }^{1}$ Guillaume Maurin ${ }^{4}$ Eric Collet,${ }^{1}$ M. Carmen Muñoz ${ }^{5}$ José Antonio Real ${ }^{6}$ and Bertrand Toudic ${ }^{1}$

${ }^{1}$ Univ. Rennes, CNRS, IPR (Institut de Physique de Rennes) - UMR 6251, F-35000 Rennes, France

${ }^{2}$ Institut des Materiaux Jean Rouxel (IMN),

Université de Nantes, UMR CNRS 6502, 2 rue de la Houssiniere, BP 32229, 44322 Nantes Cedex 3, France

${ }^{3}$ Univ. Rennes, ENSCR, CNRS, ISCR (Institut des Sciences

Chimiques de Rennes) - UMR 6226, F-35000 Rennes, France

${ }^{4}$ Institut Charles Gerhardt Montpellier, UMR 5253 CNRS UM, Université Montpellier, Place E. Bataillon, 34095 Montpellier Cedex 05, France

${ }^{5}$ Departamento de Fisica Aplicada, Universitat Politécnica de València, Camino de Vera s/n, E-46022 València, Spain

${ }^{6}$ Instituto de Ciencia Molecular (ICMol), Universitat de València, C/Catedrático José Beltràn Martìnez 2, 46980 Paterna (Valencia), Spain.

(Dated: March 3, 2020) 


\begin{abstract}
The MOF $\left[\mathrm{Fe}(\mathrm{tvp})_{2}(\mathrm{NCS})_{2}\right] \cdot 2 \mathrm{BzCHO}$ is demonstrated to undergo a complex sequence of phase transitions and spin crossover behavior of its constitutive $F e^{I I}$ ions upon adsorption of benzaldehyde guest molecules. Our study, combining Raman and synchrotron X-ray diffraction measurements on single crystal reveals that the conversion from the pure high spin to the pure low spin phases implies a rich sequence of intermediate phases, with symmetry breaking forming at least three different space groups. These different symmetry involve spin-state ordering, ligand ordering and guest ordering, interpreted by combining magnetic susceptibility, Raman measurements, Density Functional Theory and force field Molecular Dynamics calculations.
\end{abstract}

\title{
I. INTRODUCTION
}

Phase transitions are almost ubiquitous in matter. Some materials have been recently created to enhance the related structural changes such as 2D and 3D coordination polymers. In particular, coordination polymers with permanent porosity, the so-called metal-organic frameworks (MOFs), have acquired special relevance nowadays. They are constituted of metal ions or metal-ion clusters acting as nodes linked together through organic ligands. Playing with the connectivity of the nodes and the size and shape of the linkers a huge variety of networks with desired topologies and porosities has been reported in the last two decades fuelled by their potential applications in gas adsorption, separation, catalysis, drug delivery, sensing, etc [1-3]. The porous character of the MOFs allows the confinement of guest molecules, which act on the pore shape/opening and consequently on the symmetry of the structure $[4,5]$. Temperature and pressure are the most common control parameters. Transition-metal complexes with electron configuration $d^{4}-d^{7}$ may for instance exhibit bistability between two molecular states with a low-spin (LS) or a high-spin (HS) electronic and structural configurations with distinct associated entropy and size of the molecule [6, 7]. Therefore, external stimuli like temperature, pressure or light may balance the relative stability of HS and LS states and, in solids, cooperative phase transitions between LS and HS states may occur. This spin-crossover ( $\mathrm{SCO}$ ) phenomenon is accompanied by a change in the magnetic susceptibilities, the electronic absorption and vibrational spectra, as well as

*celine.mariette@univ-rennes1.fr 
metal to ligand distances and the volume of the crystal.

Recently, complex stepwise SCO phenomena aroused a great attention in the fields of chemistry and solid state physics [8-15]. Combining both MOFs and SCO properties greatly enhance the richness in terms of instabilities both from the electronic and structural points of view, as reported recently. [16-22]

The influence of a series of guest molecules, such as benzonitrile, nitrobenzene, benzaldehyde, acetonitrile/water, dimethylsulfoxide/water, on the SCO properties of this MOF-SCO was investigated more recently [16]. It was shown through the thermal dependence of $\chi_{M} T$ $\left(\chi_{M}=\right.$ molar magnetic susceptibility, $T=$ temperature $)$ that the $\left[F e(t v p)_{2}(N C S)_{2}\right]_{n}(t v p$ $=\operatorname{trans}(4,4$-vinylenedipyridine) $)$ system with no guest remains in the high spin state (HS, $\mathrm{S}=2$ ) down to low temperature. Interestingly, the inclusion of guests induces various types of $\mathrm{SCO}$ behavior towards the low spin (LS, $\mathrm{S}=0)$ state. Let us mention that, when included, these guest molecules remain in the pores during all the temperature scans from $77 \mathrm{~K}$ to room temperature. The phase transitions have strong signatures in differential scanning calorimetry measurements (DSC)[16].

When the guest molecule is benzaldehyde $\left(\mathrm{C}_{6} \mathrm{H}_{5} \mathrm{COH} ; \mathrm{BzCHO}\right)$, the $\chi_{M} T$ v.s. $\mathrm{T}$ plot probing the fraction of HS species incorporated in this MOF, shows an apparent continuous evolution between $160 \mathrm{~K}$ and $220 \mathrm{~K}$, similar to conventional spin-crossover behavior, with an intriguing change of slope around $170 \mathrm{~K}$ (figure 1). Such a change of slope is unexpected for materials undergoing so called "gradual" thermal conversions, as the usually associated spin crossover is simply described by the Boltzmann population of HS and LS states, and where the HS state of higher entropy is gradually populated as temperature increases. Differential calorimetry measurements revealed anomalous variation of the molar specific heat in the temperature range where magnetic susceptibility evolves rapidly [16]. Here we reveal a hidden sequence of symmetry breakings during the thermal spin state conversion, due to the concerted electronic conversion and structural order of HS and LS spin states in the host MOF-SCO, as well as the ordering of guests.

\section{EXPERIMENTAL APPROACH}

We used synchrotron X-ray diffraction for characterizing the different structural phases of the MOF-SCO, to determine the long-range order and the symmetry lowering as function 
a)

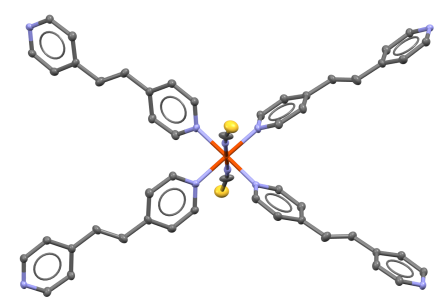

b)

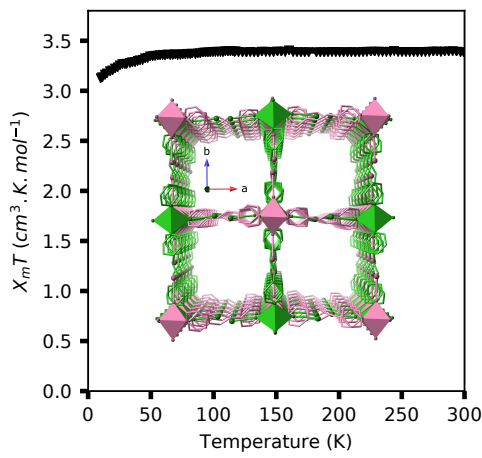

c)

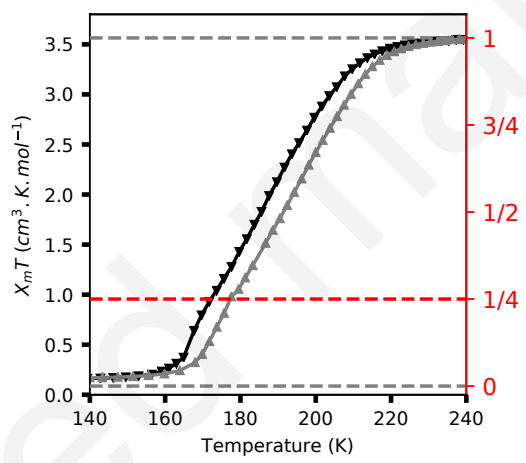

FIG. 1. a) Molecular model used for DFT calculations(red: Fe, blue: N, grey: C, yellow: S). b) $\chi_{M} T$ product $v s$ temperature of the empty host system upon cooling. Insert: Representation of the host structure showing the 3D interpenetrated MOF framework of the $\left[\mathrm{Fe}(t v p)_{2}(\mathrm{NCS})_{2}\right] \cdot 2 \mathrm{BzCHO}$. The $2 \mathrm{BzCHO}$ guest is omitted for clarity. c) $\chi_{M} T$ product $v s$ temperature of this host/guest system upon warming (in grey) and upon cooling (in black). Right red axis indicates the corresponding $x_{H S}$ ratio, assuming a residual paramagnetic contribution equal to $0.025 \mathrm{~cm}^{3} . K . \mathrm{mol}^{-1}$ $[16]$.

of the temperature. To unravel a complex ordering, high-flux and high-resolution diffraction measurements were performed on the beamline SNBL at the ESRF synchrotron, with a fixed wavelength of $\lambda=0.69730 \AA$. Measurements were done with a single $\phi$-axis rotation goniometer over 360 degrees with a 0.2 degree step. A Pilatus $2 \mathrm{M}$ detector with very 
low noise, was placed at $146.2 \mathrm{~mm}$ from the sample. Subsequently, the diffraction planes were reconstructed using CrysAlisPRO [23] software from Rigaku Oxford Diffraction. Complementary laboratory measurements were performed on a SuperNOVA diffractometer of Agilent Technologies, with a highly sensitive EosS2 CCD detector and microfocus copper source $(\lambda=1.54184 \AA$ A ). The latter consisted in short temperature measurements (of c.a. $10 \mathrm{~min}$ each) with $2 K$ steps at $120 \mathrm{~K} / \mathrm{h}$ rate between $140 \mathrm{~K}$ and $240 \mathrm{~K}$ to ensure a slow but homogeneous cooling/heating within the crossover temperature range. In both cases, the crystals were cooled respectively with flowing nitrogen 700 and 800Plus series cryostats from Oxford Cryosystems.

Molecular dynamics (MD) simulations were carried out to explore the structure of guest molecules confined within the MOF-SCO. MD simulations consist in the numerical integration of the equations of motions based on the use of interatomic potentials allowing the calculation of the atomic force generated. Initial configurations of the guest molecules were generated by means of Monte Carlo (MC) simulations performed in the grand canonical ensemble. More computational details are provided in the Appendix A.

Raman spectroscopy was performed to study selectively the vibrational properties of the high spin and low spin species. The measurements were done using a backscattering micro-Raman spectrometer (T64000 from Jobin Yvon) with helium-neon laser (633 nm) excitation. The spectral resolution for the recorded Stokes-side Raman spectra were set to $2 \mathrm{~cm}^{-1}$ (this resolution was achieved by the use of 1800 grooves $/ \mathrm{mm}$ gratings) in order to collect maximum signal quality. Raman spectra of single crystalline $\left[\mathrm{Fe}(\mathrm{tvp})_{2}(\mathrm{NCS})_{2}\right]$. $2 \mathrm{BzCHO}$ were recorded in the temperature range of 100-270 K. Low-temperature Raman studies were conducted using a $N_{2}$-gas flow cryostat. The incident laser beam was 0.8 $\mathrm{mW}$ power. The laser intensity was optimized to avoid crystal damaging which might be associated with any laser induced effect. The peaks were fitted as Lorentzian, in order to follow the thermal behavior of peaks position, width and intensity. A global normalization of the spectra was applied to account for the strong absorption decrease with the spin state fraction.

We used Density Functional Theory (DFT) calculation to obtain the frequencies modes of the molecular model $\left[\mathrm{Fe}(\mathrm{tvp})_{2}(\mathrm{NCS})_{2}\right]$ in order to interpret our experimental Raman data. From the crystal structure we had to build a molecular model consisting of one iron site linked with four groups and two NCS (figure 1a)). The starting atomic positions were the 
ones obtained by X-ray diffraction structure solution at $250 \mathrm{~K}$, as discussed in part III. The computations start with a geometry optimization, aiming at finding the atomic coordinates which minimize the total energy of the molecule. Once the optimized geometry found, the computation of the normal modes of vibration can be carried out. We used the MPW1PW91 functional [24] which has proven to lead to reliable results for molecular ground state and excited states properties. The standard 6-31G* atomic basis set has been used whereas all computations have been carried out using the Gaussian 09 package [25] The MPW1PW91 calculations usually gives frequencies for the vibrational modes, which are reasonable but typically differ from the experimentally observed frequencies by approximately $20-30 \mathrm{~cm}^{-1}$. In most cases all predicted vibrations are shifted in one direction, thus sometimes empirical scaling factors are used in order to match the experimentally observed frequencies [26]. The scaling factors are characteristic of the applied DFT functional and basis sets and are available from the Computational Chemistry Comparison and Benchmark DataBase [27]. This values are valid for the highest frequency modes but usually higher and close to 1 for the lower frequencies. In our case, we applied a scaling factor of 0.98 for the frequency range $1000-1300 \mathrm{~cm}^{-1}$ shown in the present manuscript [28]. This scaling factor corrects partly for the neglect of anharmonic corrections [29-31]. Most importantly, the used program allows us to fix the spin state of the system, so that in our case the calculations were conducted in both high spin and low spin states. In the HS state, the computed spin population of the iron atom is found equal to 3.852 close to the ideal values 4 , some spin delocalization occurring in the system.

\section{RESULTS}

\section{A. X-Ray Structural Characterization of the Phase Transitions}

The series of compounds generically formulated as $\left[\mathrm{Fe}(\mathrm{tvp})_{2}(\mathrm{NCS})_{2}\right] \cdot$ Guest is made up of 2D $\left[\mathrm{Fe}(\mathrm{tvp})_{2}(\mathrm{NCS})_{2}\right]_{n}$ layers where the crystallographically unique $F e^{I I}$ pseudo-octahedral sites are equatorially coordinated by $4 \mathrm{tvp}$ ligands each one bridging $2 F e^{I I}$ centers. The axial positions of $F e^{I I}$ are occupied by two terminal $N C S^{-}$anions (see figure 1 a)). The polymeric layers define wide $\left.\left[\mathrm{Fe}_{4}(t v p)_{4}\right]_{n}\right]$ square windows that allow the interpenetration of an equivalent set of $2 \mathrm{D}\left[\mathrm{Fe}(\mathrm{tvp})_{2}(\mathrm{NCS})_{2}\right]_{n}$ orthogonally oriented with respect to the 


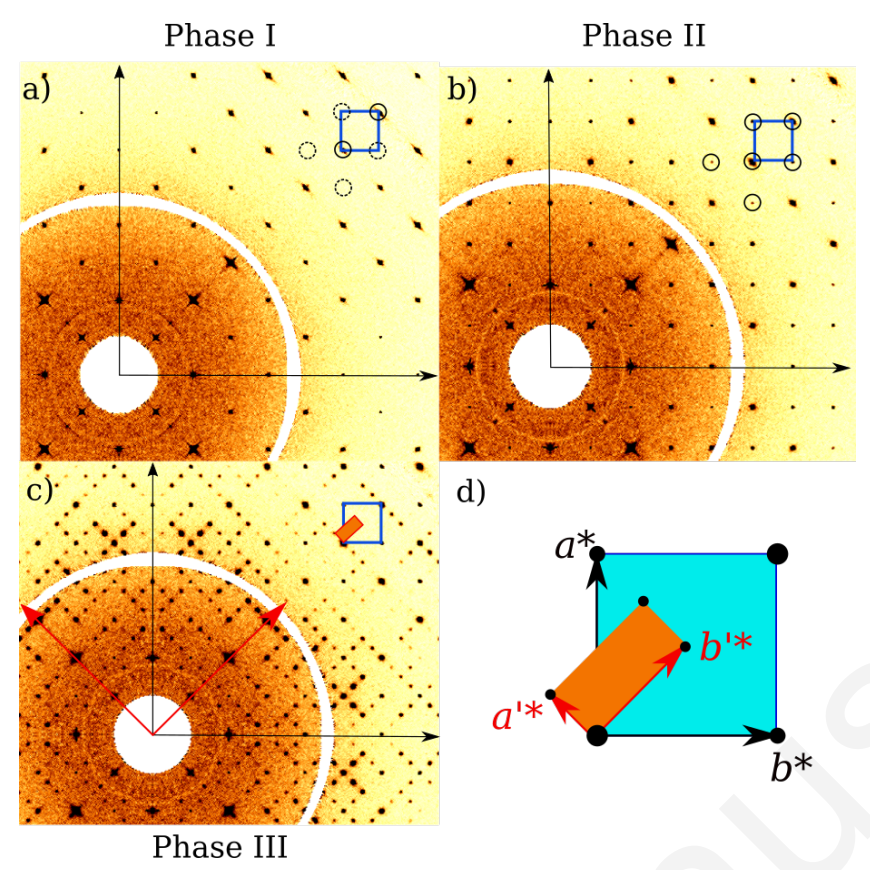

FIG. 2. The diffraction image of the $\left(\mathbf{a}^{*}, \mathbf{b}^{*}\right)$ plane in three different phases: a) Phase I, measured at $T=250 K, \mathrm{~b})$ Phase II ,measured at $T=210 \mathrm{~K}$ with the appearance of $\mathrm{h}+\mathrm{k}$ odd Bragg peaks associated with the loss of a gliding plane. The unit cell of these high symmetry phases is shown in blue. c) Phase III, measured at $T=195 \mathrm{~K}$ : the superstructure Bragg peaks are described with the new unit cell shown in red, $\left.\mathbf{a}^{*}=\left(\mathbf{a}^{*}-\mathbf{b}^{*}\right) / 4, \mathbf{b}^{*}=\left(\mathbf{a}^{*}+\mathbf{b}^{*}\right) / 2\right)$. d) Schematic representation of the reciprocal cells showing the axis changes between phase II and phase III.

former thereby defining large square-sectional channels whose diagonals span c.a. $21 \AA$ where the guest molecules are docked (figure $1 \mathrm{~b}$ )). In particular, the structure of the loaded $\left[\mathrm{Fe}(\mathrm{tvp})_{2}(\mathrm{NCS})_{2}\right] \cdot 2 \mathrm{Bz} \mathrm{CHO}$ is tetragonal with $a=b=15.95 \AA$ and $c=15.51 \AA$, at $T=250 \mathrm{~K}$. The disorder of the guest molecules remains always to important to allow any structural resolution for these molecules. This important point will be extensively discussed later on in the article. The diffraction conditions of presence of Bragg peaks lead to the space group $P 4 / n 2_{1} / c 2 / c$ for this phase I (see figure 2a), in agreement with the published space group for this compound [16]. The different structural phases have been determined by single crystal X-ray diffraction revealing two structural thermal phase transitions as discussed below. Consequently, we performed complete data acquisitions in the different phases in order to determine the space group for each phase. Figure 2 displays the observed diffracted intensity reconstructed within the selected $\left(\mathbf{a}^{*}, \mathbf{b}^{*}\right)$ reciprocal plane. Phase II 
is characterized by the disappearance of glide planes, as evidenced in particular by the appearance of (hk0) Bragg peak with $\mathrm{h}+\mathrm{k}$ odd in $\left(\mathbf{a}^{*}, \mathbf{b}^{*}\right)$ plane, at $T=210 \mathrm{~K}$ in figure $2 \mathrm{~b})$. At this temperature the crystal system remains tetragonal with $a=b=15.77 \AA$ and $c=15.54 \AA$. The study of systematic extinctions is consistent with the tetragonal space group $P \overline{4} 2_{1} c$ for this phase II.

The diffracted signal in the reciprocal planes at $T=195 \mathrm{~K}$ reveals a much denser set of Bragg peaks in the $\left(\mathbf{a}^{*}, \mathbf{b}^{*}\right)$ plane as shown in the figure $\left.2 \mathrm{c}\right)$. Superstructure Bragg peaks appear at position multiple of $1 / 4 \mathrm{a}^{*}$ or $1 / 4 \mathrm{~b}^{*}$. Two possible solutions may be considered. The first one is a tetragonal phase with a cell multiplicity of 16 ( 4 along a and b). However, the Bragg peaks which should be related to a combined quadruplicating of the cell along $\mathbf{a}$ and $\mathbf{b}$, i.e. simultaneously at $1 / 4 \mathbf{a}^{*}$ and $1 / 4 \mathbf{b}^{*}$, are not present, as expected in such a case. The second solution, which does not require appearance of Bragg peaks simultaneously at $1 / 4 \mathbf{a}^{*}$ or $1 / 4 \mathbf{b}^{*}$ corresponds the formation of two symmetry related orthorhombic domains, characterized by the reciprocal vectors $\mathbf{a}^{*}=\left(\mathbf{a}^{*}-\mathbf{b}^{*}\right) / 4, \mathbf{b}^{*}=\left(\mathbf{a}^{*}\right.$ $\left.+\mathbf{b}^{*}\right) / 2$ (see figures $2 \mathrm{c}$ ) and $\mathrm{d}$ )). In the real space this corresponds to the orthorhombic unit cell defined by $\mathbf{a}^{\prime}=\mathbf{a}+\mathbf{b}$ and $\mathbf{b}=2 \mathbf{a}-2 \mathbf{b}$. Let us mention that no Bragg peak splitting is observed and so no deviation from the tetragonal metric is apparent within the resolution of our study. The cell parameters at $\mathrm{T}=195 \mathrm{~K}$ refined considering these two orthorhombic domains are $: a^{\prime}=22.03 \AA, b^{\prime}=44.06 \AA$ and $c^{\prime}=15.57 \AA$, i.e. $a^{\prime}=2 b^{\prime}$ within our experimental resolution. The highest symmetry space group for this phase III is found to be Pnn2. The phase transition from the tetragonal phase II to orthorhombic phase III is therefore of ferroelastic nature.

In order to determine the transition temperatures $T_{c 1}$ and $T_{c 2}$ associated with the two symmetry breaking phase transitions, the intensity of the Bragg peaks, signing respectively the loss of the gliding planes and the multiplicity of the unit cell, were measured as a function of temperature (figure $3 \mathrm{a}$ )). This leads to the following values: $T_{c 1}=212 \mathrm{~K}$ and $T_{c 2}=201 \mathrm{~K}$. Let us note here that the tetragonal high symmetry unit cell is used as reference in the following to reveal the thermal evolution of the lattice. The evolution of the volume of this retained unit cell (figure $3 \mathrm{~b}$ )) shows strike similarity with the one of the magnetic susceptibility (figure $1 \mathrm{c}$ )), [16]). According to our measurements, it appears that the parameters $a(=b)$ of this tetragonal unit cell decreases rather continuously with a change of slope around $T=175 \mathrm{~K}$, similar to the magnetic curve (figure $1 \mathrm{c}$ )). Quite 

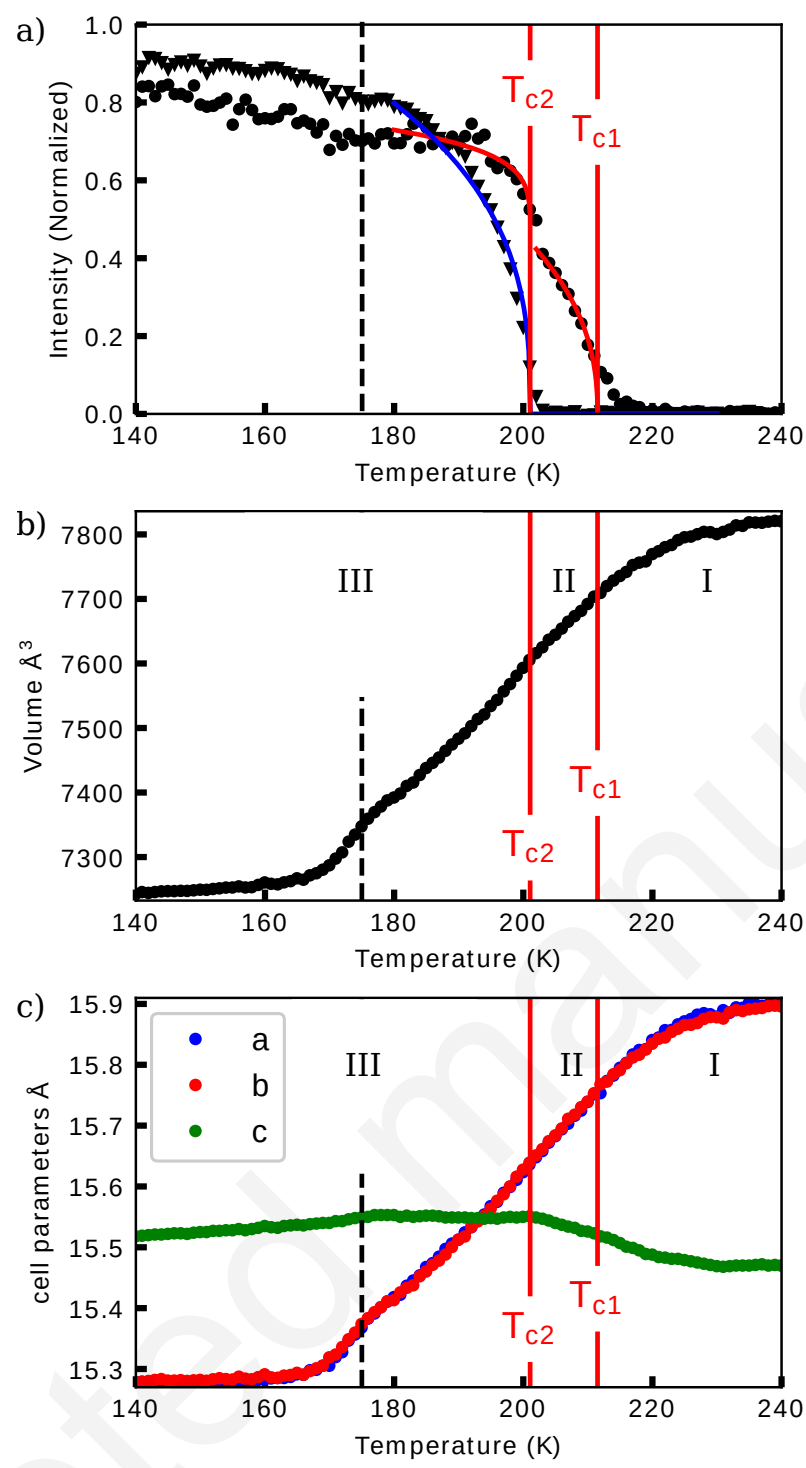

FIG. 3. a) Temperature evolution of Bragg peaks appearing in phases II below $T_{c 1}$ and III below $T_{c 2}$. In black circles, Bragg peaks appearing in phase II due to the loss of glide planes (averaged over 16 Bragg peaks, normalized), and in black triangles, superstructure Bragg peaks signing the cell multiplication in phase III (averaged over 309 peaks). (b) and (c) Temperature evolution (on cooling) of unit cell volume (c) and the cell parameters (b) in the unit cell basis from the high temperature tetragonal phase. $T_{c 1}$ and $T_{c 2}$ indicated as red vertical lines are extracted from intensities evolutions shown in (a). Dashed vertical black line indicate the anomaly correlated with the one observed in magnetic measurements. 
a)

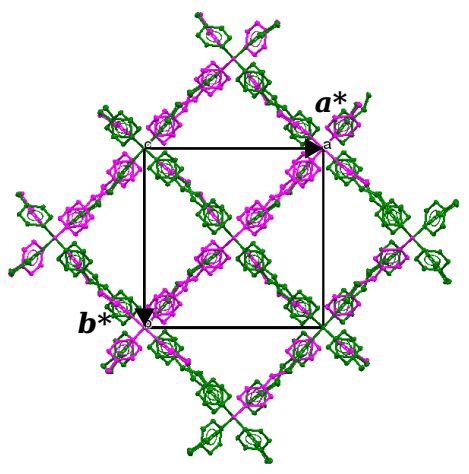

C)

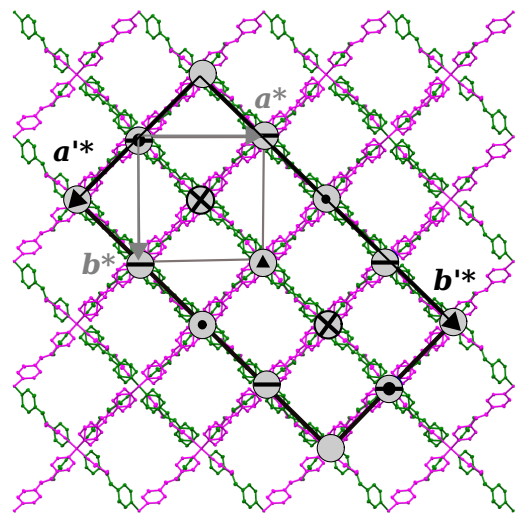

b)

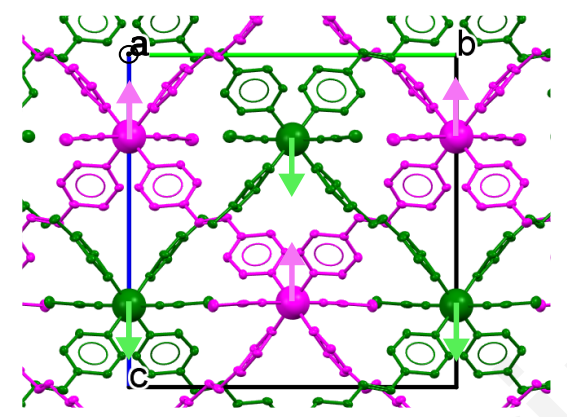

d)

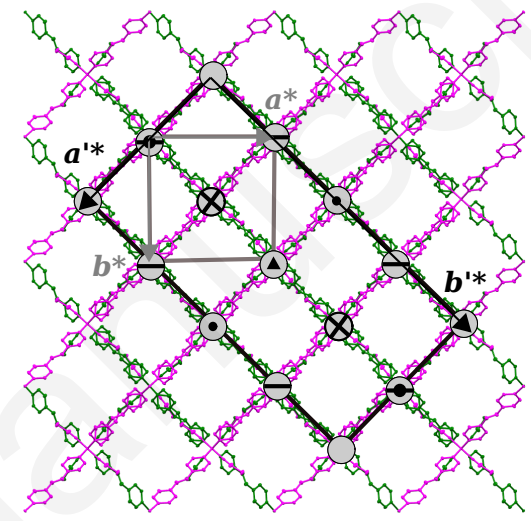

FIG. 4. Schematic representation of the host structures in the low symmetry phases. The guest molecules are omitted for clarity. a) Phase II: view in the (a, b) plane. b) Phase II: view in the (b, c), showing the two interpenetrated lattice (in grey and magenta), displaced in opposite direction from the high symmetry position (1/4 and $3 / 4$ along $\mathbf{c})$. c) Phase III: view in the (a, b) plane showing the large 4 by 2 unit cell (the high symmetry tetragonal cell is shown in light grey for reference). Independent Fe sites are marked with different symbols. d) Phase III: Possible spin state ordering compatible with the symmetry depicted in c) and the 1/4 High Spin fraction on the plateau at $\mathrm{T}=175 \mathrm{~K}$.

differently, the value of the $c$ parameter of this tetragonal unit cell slightly increases in the temperature range from $T_{c 1}=212 \mathrm{~K}$ to $T_{c 2}=201 \mathrm{~K}$. The $c$ parameter remains then almost constant down to $T=175 \mathrm{~K}$ and then follows a linear thermal contraction behavior below this temperature.

The peculiar feature of this temperature, which does not correspond to any symmetry breaking, will be further discussed later on. The spin conversion is thus associated with important structural reorganizations of the $\left[\mathrm{Fe}(\mathrm{tvp})_{2}(\mathrm{NCS})_{2}\right] \cdot 2 \mathrm{BzCHO}$ lattice. The high 
symmetry phase $\mathrm{I}$ is quadratic $P 4 / n 2_{1} / c 2 / c$. In the phase, all the Fe atoms are in the HS state and symmetrically equivalent. The first structural instability corresponds to a group/subgroup phase transition associated with the loss of glide planes, leading to phase II with quadratic space group $P \overline{4} 2_{1} c$ (figure 4 a)). The loss of a glide plane along the c direction allows the Fe sites to move slightly away from the position $z=1 / 4$ (or $z=3 / 4$ ) along $\mathbf{c}$, the two interpenetrated sublattices moving in opposite directions (see figure $4 \mathrm{~b}$ )). This results in the anomalous temperature behavior of the c parameter described above. However in this phase II all the Fe sites remain symmetry equivalent and therefore the thermal spin conversion corresponds to a gradual SCO. The second phase transition at $T_{c 2}=201 K$ generates a new crystallographic phase III, found to be orthorhombic Pnn2 and associated with a cell multiplication. This orthorhombic phase group is a subgroup of the quadratic one of phase II. This supercell contains by symmetry six inequivalent Fe sites, as schematically shown in figure $4 \mathrm{c}$ ). The complexity of the structure (low symmetry, large unit cell, domains) precluded any reliable structural model refinement.

In this phase III, the thermal spin conversion shows a particular behavior, marked by a small plateau at $x_{H S}=1 / 4$ and associated to clear conversion step around $\mathrm{T}=170 \mathrm{~K}$ towards $x_{H S}=0$. This plateau is also observed in the temperature dependence of the lattice parameters (figure $3 \mathrm{~b}$ ) and c)). In the present case, the occurrence of a plateau at $x_{H S}=1 / 4$ in the low symmetry phase III can be explained by the formation of a periodic spin-state concentration waves, forming during Devil's staircase-type spin-crossover. The symmetry breaking that generates inequivalent Fe sites is associated with long-range order of HS and LS states on the sites [8-15]. We represent schematically in figure $4 \mathrm{~d}$ ) one possible order of HS and LS molecular states, which is allowed by the orthorhombic Pnn2 space group of phase III.

\section{B. Spin crossover and Structural changes: Density Functional Theory calculations} and Raman spectroscopy

DFT calculations were first performed for determining the local geometry in both HS and LS states, considering the molecular model focusing on the host structure (figure $1 \mathrm{a}$ ), see also experimental paragraph). Regarding the simulated DFT Raman spectra, we built and computed a large molecular model able to describe accurately the relevant internal modes 

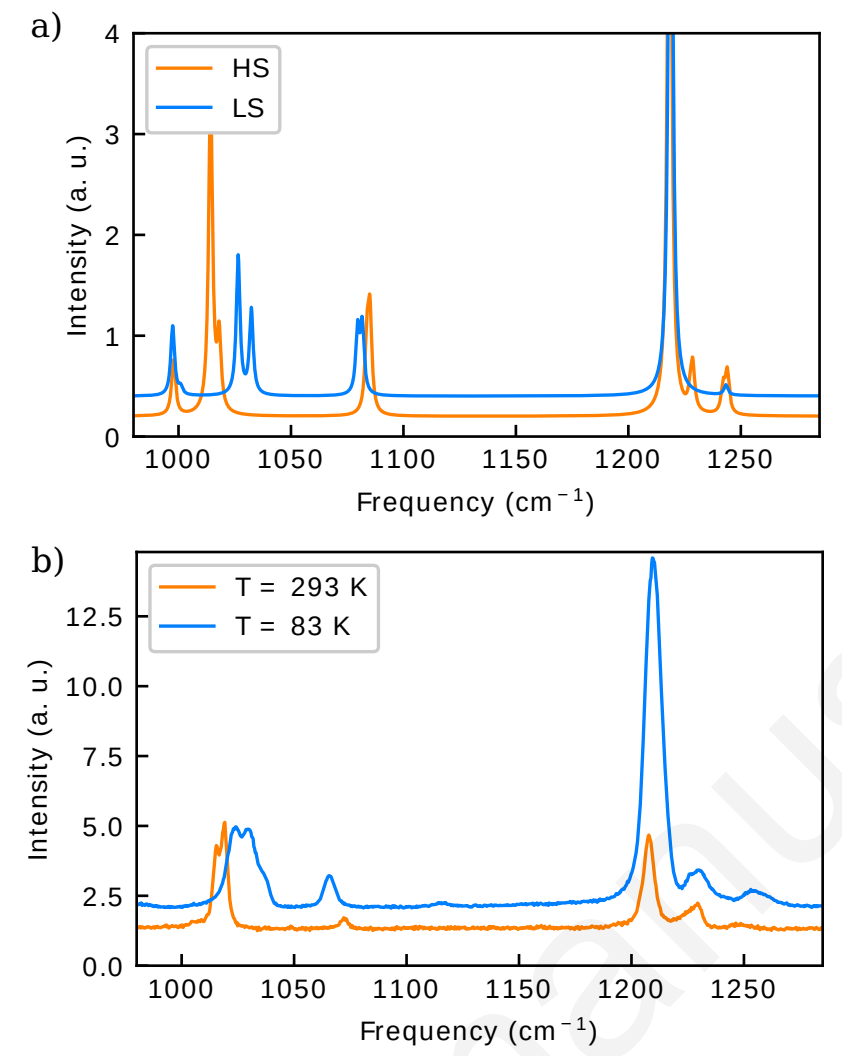

FIG. 5. a) calculated Raman spectra within the frequency $950-1250 \mathrm{~cm}^{-1}$ in the High Spin (orange) and Low Spin state (blue); b) measured Raman spectra in the corresponding frequency range at $\mathrm{T}=270 \mathrm{~K}$ and $\mathrm{T}=100 \mathrm{~K}$.

of vibrations of the system.Optimized values of the interatomic distances were extracted and compared to the experimental ones present in the crystal structure. The $F e-N$ bond lengths of the optimized geometry are very close to the experimental X-ray diffraction results both in the HS and LS states. In the HS state, the calculated and the experimental distances $F e-N_{N C S}$ and $F e-N_{t v p}$ are respectively $2.13 \AA$, compared to $2.10 \AA$ and $2.20 \AA$, compared to $2.21 \AA$. In the LS state, the calculated and experimental distances $F e-N_{N C S}$ and $F e-N_{t v p}$ are respectively $1.93 \AA$, versus $1.93 \AA$ and $2.00 \AA$, versus $2.00 \AA$. The expansion in the HS state is expected, due to the volume increase of the divalent Fe coordination sphere arising from the occupation of anti-bonding $e_{g}^{*}$ states with two unpaired electrons, as in the almost octahedral ligand field the electronic distribution changes from LS $\left(t_{2 g}^{6} e_{g}^{0}\right)$ to HS $\left(t_{2 g}^{4} e_{g}^{2}\right)$. This agreement underlines the reliability of the DFT approach and confirms the validity of the subsequent dynamical calculations. 

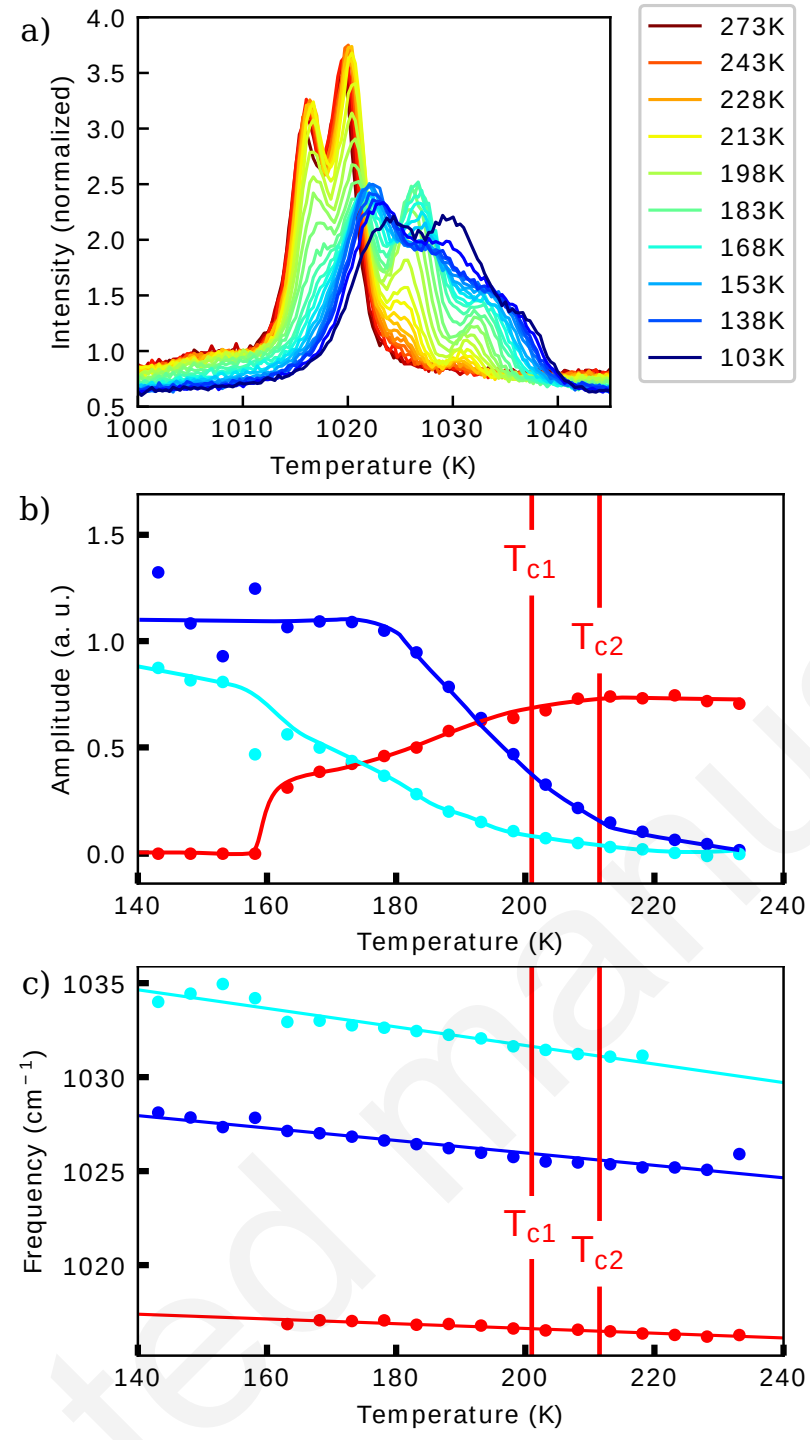

d)

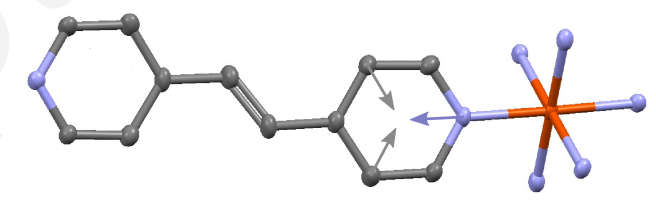

FIG. 6. a) Temperature evolution of Raman spectra measured between $1000-1045 \mathrm{~cm}^{-1}$; b) Temperature evolution of the related peak amplitudes. c) Temperature evolution of the frequency of three Raman peaks, extracted from a fit of pattern a) with a sum of gaussian peaks; in b) and c) the vertical red lines points $T_{c 1}=212 \mathrm{~K}$ and $T_{c 2}=201 \mathrm{~K}$ as extracted from X-ray diffraction study. d) Schematic representation of the most intense calculated Raman mode in the corresponding wavenumber range. The vectors indicate the direction of the force momentum (red: Fe, blue: N, grey: C, hydrogen atoms are omitted for clarity). 
a)
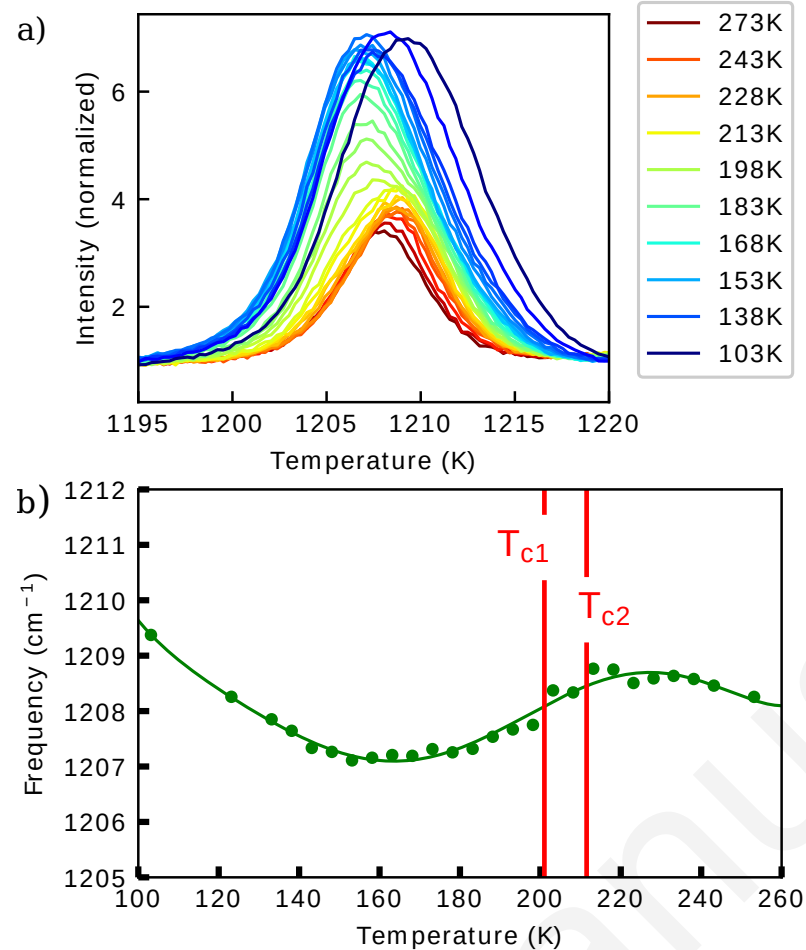

C)

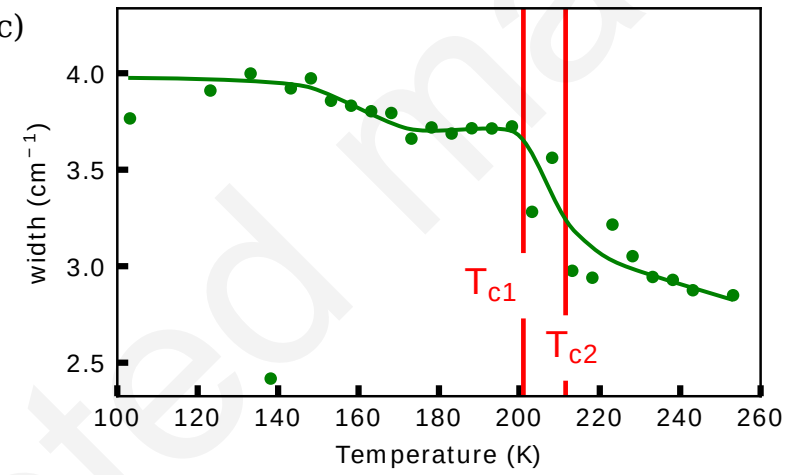

d)

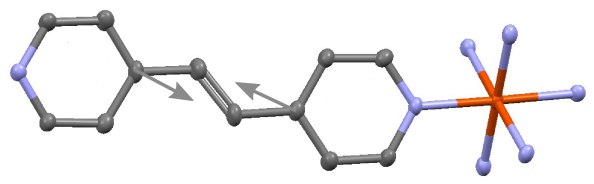

FIG. 7. a) Temperature evolution of Raman spectra measured between $1195-1220 \mathrm{~cm}^{-1}$. B) Temperature evolution of the frequency of the peak (extracted from a fit with a gaussian function) showing a softening around the phase transition, and c) of the corresponding peak width $(\sigma)$; in b) and c) the vertical red lines points $T_{c 1}=212 \mathrm{~K}$ and $T_{c 2}=201 \mathrm{~K}$ as extracted from X-ray diffraction study. d) Schematic representation of the calculated Raman mode at $1205 \mathrm{~cm}^{-1}$ in the HS state. The vectors indicate the direction of the force momentum (red: Fe, blue: N, grey: C, hydrogen atoms are omitted for clarity). 
The optimized molecular geometry is used to extract the vibrational properties (see methods paragraph). Figure 5 a) shows the calculated Raman spectra in the HS and LS states. They have to be compared with the experimental measurements shown in the figure $5 \mathrm{~b}$ ), performed at $\mathrm{T}=293 \mathrm{~K}$ for the HS state and $\mathrm{T}=83 \mathrm{~K}$ for the LS state. For better comparison, the average measured peak width $\left(2 \mathrm{~cm}^{-1}\right)$ was used to plot the theoretical profiles based on the calculated Raman frequencies. A good agreement is found between the experimental and calculated frequencies of the Raman active modes, as well as relative intensities. A major information given by DFT calculations is the polarization of each mode.

In this article, we will focus on two narrow frequency ranges around $1020 \mathrm{~cm}^{-1}$ and $1200 \mathrm{~cm}^{-1}$ (see figures 6 and 7). The Raman measurements around $1020 \mathrm{~cm}^{-1}$ (figure 6) provide a selective way to study separately the fraction of molecule in the HS state $\left(x_{H S}\right)$ and in the LS state $\left(x_{L S}\right)$ from the intensity of the LS and HS Raman bands, since their spectral signatures are significantly different. According to polarization calculations, the modes in this region corresponds to breathing of the benzene ring attached to the Fe sites through the $F e-N$ bond (figure $6 \mathrm{~d}$ )). It will thus intuitively be very sensitive to the spin state, due to the change of the $F e-N$ distance. The temperature evolution of the spectra in the frequency range $1000-1045 \mathrm{~cm}^{-1}$ is shown in the figure $6 \mathrm{a}$ ). The HS spectra as a double peak centered around $1015-1020 \mathrm{~cm}^{-1}$. The two frequencies being very close to each other, these two peaks are not separated in the theoretical spectra as plotted here. The appearance of the LS peaks at higher frequency signs the beginning of the spin state conversion (see figure $6 \mathrm{~b}$ )). It is found around $\mathrm{T}=220 \mathrm{~K}$ in agreement with the magnetic measurement. The end of the spin state conversion is marked by the disappearance of the HS peak (around $1015 \mathrm{~cm}^{-1}$ ). It is found at $\mathrm{T}=160 \mathrm{~K}$, which again agrees with the $\chi_{M} T$ curve .

No anomaly is observed at $T_{c 1}$ nor $T_{c 2}$, and the frequencies of the selected bands follow the expected linear increase when temperature is lowered (figure 6c)). This is not the case in the second frequency window we present $\left(1195-1220 \mathrm{~cm}^{-1}\right)$, where the mode is associated with squeezing of the $t v p$ ligand (see figure $7 \mathrm{~d}$ )). The main Raman mode around $1205 \mathrm{~cm}^{-1}$, reveals an anomalous dependence of its frequency versus temperature (figure 7a) and b)). Instead of the expected hardening, the mode softens between $T_{c 1}$ and $T_{c 2}$. This behavior is correlated with the anomalous increase of the c lattice parameter, described in paragraph III-B (figure $3 \mathrm{c}$ )). This lowering of the frequency continues to $T \simeq 160 \mathrm{~K}$, the end of the 

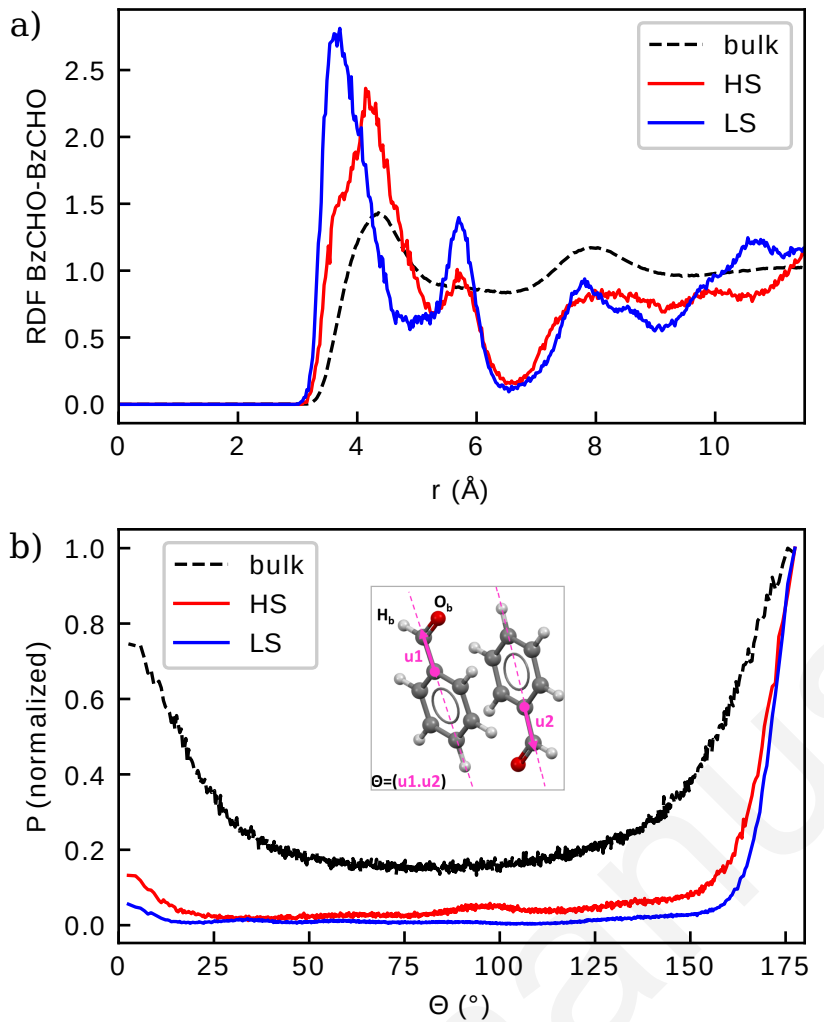

FIG. 8. a) Radial Distribution Functions between the centre of mass of the guest molecules. b) Angular distribution of the angle between $u_{1}$ and $u_{2}$ for both LS and HS forms (normalized to the value at $180 \mathrm{deg})$.

spin state conversion. The linear increase of the frequency of the mode is observed below $T=160 K$ as expected when cooling down. We note that going through the phase transition there is an apparent peak broadening (figure $7 \mathrm{c}$ )), maybe related to the symmetry breaking, inducing the loss of degeneracy of these Raman modes.

\section{Molecular dynamics characterization of the host-guest interactions}

X-ray diffraction did not allow determining the organization of the confined guests within the host matrix, due to large disorder in the structure. To overcome this problem, force field Monte Carlo and Molecular Dynamics simulations were carried out to get microscopic insights of the host-guest and the guest-guest interactions. Computational details are provided in the annex.

Radial distribution functions (RDF) between the centers of mass of the benzaldehyde 


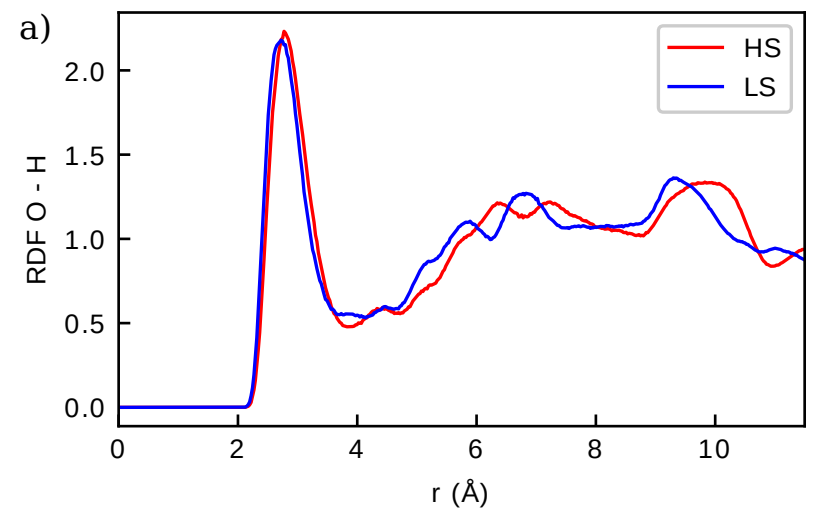

b)

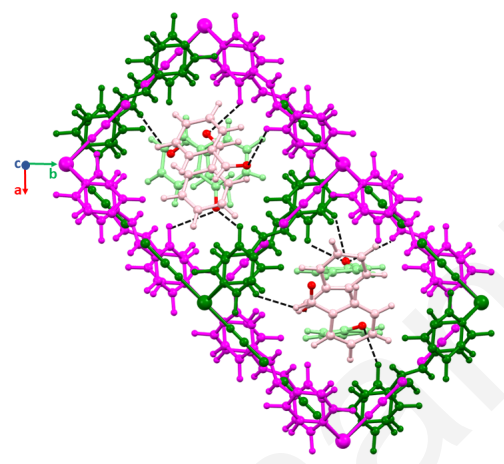

FIG. 9. a) Radial distribution functions between the oxygen atom of the benzaldehyde and the hydrogen atom bound to the carbon atom of the MOF-SCO material. b) Illustration of characteristic $\mathrm{CH} \cdots \mathrm{O}$ of the first peak of $\mathrm{RDF}$, showing one representative configuration of the host/guest interactions implying hydrogen bonds.

molecules in confined and bulk phases for a loading of 8 molecules per unit cell are reported in Figure 8 a). First peak of the RDF is located at $3.7 \AA$. $4.1 \AA$ and $4.2 \AA$ for LS, HS and bulk media respectively. The so-found slightly shorted guest/guest distances in both HS and LS frameworks as compared to the bulk state shed light on a specific conformation adopted by the molecules with respect to each other. To identify it we report in figure $8 \mathrm{~b}$ ) the distribution $(\mathrm{P}(\Theta))$ of the angle $(\Theta)$ between the vectors connecting the carbon atom of the benzene cycle bonded to the $\mathrm{COH}$ group and the carbon atom of $\mathrm{COH}$ group (see the inset of figure $8 \mathrm{~b}$ )). Clearly, a single orientation is privileged (antiparallel) in both HS and LS MOF-SCO materials, whereas both parallel and antiparallel orientations are observed in the bulk phase. Therefore, we can conclude that the benzaldehyde molecules adopt a sandwich conformation, which is in favor of the $\pi-\pi$ interactions. Interestingly, figure 8 b) shows that other conformations are almost improbable whereas in the bulk phase their 


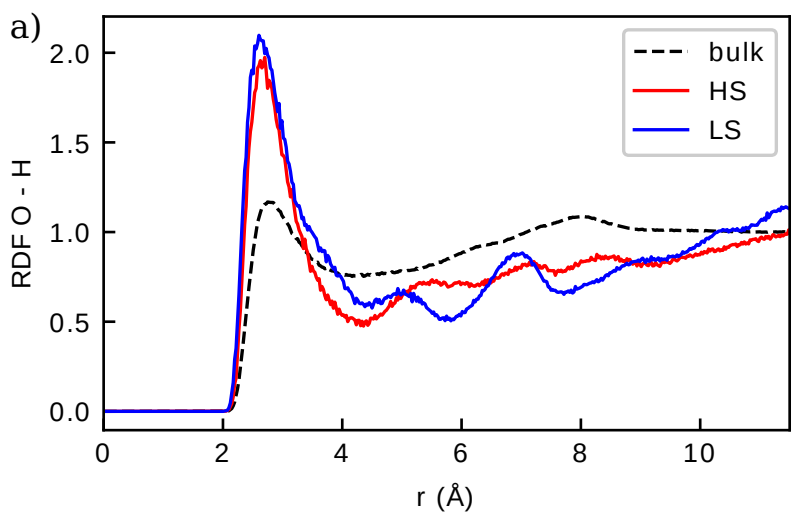

b)

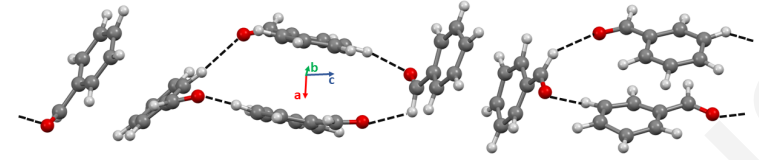

c)

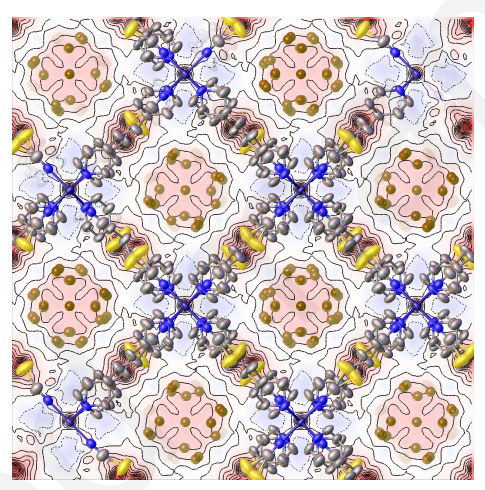

FIG. 10. a) Radial Distribution Functions between the oxygen atom of one guest molecule of $\mathrm{BzCHO}$ and the hydrogen atom of another guest molecule. b) Illustration of characteristic $O \cdots H$ of the first peak of RDF, showing one over many configurations of the arrangement of the guest dimers along the channel direction (red: O, grey: C, white: H). c) High temperature structure as solved based at $\mathrm{T}=250 \mathrm{~K}$ on $\mathrm{X}$-ray synchrotron data revealing the absence of apparent ordering of the guest molecules. The gold peaks shows residual electronic density in the middle of the channels, indicating the possible position of the disordered guest molecules.

probabilities are not negligible. The size and shape of the channels of MOF-SCO probably forces the molecules to self-assemble in this conformation.

The host-guest interactions were also investigated by extracting the RDF between the oxygen atoms of the benzaldehyde and the hydrogen atoms of MOF-SCO. As shown in Figure 9 a) and 9 b), this interaction seems to be energetically favorable with an associated 
relatively short separating distance of $2.5 \stackrel{\circ}{A}$. Let us mention that all RDFs between atoms of benzaldehyde and MOF-SCO have been calculated and we found that the most favorable interaction was between these two series of atoms linking host and guest molecules.

To analyze in more detail the organization of the $2 \mathrm{BzCHO}$ pairs in the channels, the $\mathrm{RDF}$ between the oxygen and the hydrogen atoms of the $\mathrm{OH}$ groups of guest molecules are calculated and reported in Figure 10 a). The first peak of the RDF, located around $2.3 \AA$, is of the same order of magnitude than hydrogen bonds. A representative guest configuration along the channel direction is shown in figure $10 \mathrm{~b}$ ). This local inter-guest organization might preclude any long-range ordering within the pores, and result to the disordered distribution observed by x-ray diffraction at all temperature (see figure $10 \mathrm{c})$ ). The whole organization of the $\mathrm{BzCHO}$ molecules is the result of a combination of hydrogen bonding, $\pi-\pi$ interactions and a confinement effect. These MD calculation provide essential information for characterizing the dimer arrangement of the guest molecules within the MOF and about the nature of the driving force ruling the anchoring of guest molecules on the wall of MOF-SCO, as the importance of the hydrogen bond between host and guest is underlined.

\section{CONCLUSION}

In this work, we considered a MOF which potentially may present a spin crossover (SCO) conversion. Spin crossover materials are characterized by their important induced volume changes between the High Spin and the Low Spin states. The SCO phenomenon is in many cases isostructural, i.e. it generally occurs without symmetry change and in a single step. The associated conversion from HS to LS is accompanied by a contraction of the Fe-N bonds due to the depopulation of the less bonding eg states and the modification of the ligand field. In other cases, symmetry changes also occur during the conversion from HS to LS: it may be due to long-range order of HS and LS states, to torsion of the ligand... Such symmetry breakings are often coupled to the intra-molecular reorganizations due to the change of crystal field. This is also true in the present case: we observe an average contraction of the Fe-N bonds of $2.13 \AA / 2.10 \AA$ in the HS state to $1.93 \AA / 2.00 \AA$ in the LS state also associated with a shift of the Raman bands due to the change of ligand field. However, due to intermolecular interactions related to lattice contraction during the conversion from HS to LS phases, mediated by the guest and/or the host, other phases occurs as the ligand 
field reorganization couples to symmetry changes. According to magnetic susceptibility measurements, the retained MOF-SCO Fe(tvp $)_{2}(\mathrm{NCS})_{2}$ does not present any spin crossover on cooling down when free of guests. The co-crystallization with different guests allows such spin crossover phenomenon. The retained guest is here benzaldehyde. As observed in several MOF crystals, the role of the guest is to favor a reduction of the volume of the host due to attractive guest-host-guest interactions. Molecular dynamics shows that there are two guest molecules per cage and that these molecules are oriented essentially anti-parallel. These molecular dynamics calculations reveal hydrogen bonds between the guest and host tending to bring them closer. Guest-guest hydrogen bonds also exist along kind of chains within the crystal. These interactions may appear in competition with the periodic ones within the cages and as a consequence a strong disorder of the orientation of these guests come out from the diffraction studies at all temperatures. For this retained MOF-SCO, the magnetic susceptibility measurements exhibits a rather continuous behavior. Only a slight inflexion point is reported around the value corresponding to a high spin fraction equal to $1 / 4$. The diffraction study shows that the structural changes are actually quite complex: two phase transitions corresponding to two group/subgroup symmetry lowerings are observed at temperatures where the susceptibility does not present any anomaly. On the opposite, the diffraction study reveals that no symmetry change occurs where the small plateau is observed in the magnetic susceptibility. The plateau is explained as a spin-state ordering arising from the inequivalent Fe sites generated by the second symmetry breaking. The measurements by Raman scattering allows a separate study of optical modes sensitive either to the Low Spin or the High Spin state. Density Functional Theory calculations gave key information to identify the relevant vibration modes. It allowed to reveal the anomalous behavior of the squeezing mode of the tvp ligand. As shown by molecular dynamics, this ligands are interacting with the guest molecules through hydrogen bonds. This underlines the essential role of the host-guest interactions in the symmetry breakings, and is also fully consistent with the anomalies observed on the evolution of the unit cell parameters. This work shows that the inclusion of guest molecules in host MOF materials opens a rich field of investigation in new systems presenting bistable spin states for tuning spin crossover or for generating stepwise phase transitions. 


\section{Appendix A: Computational Details}

\section{Partial Charges Calculations}

Following the procedure proposed by Chen et al., the calculation of the partial charges of both HS and LS MOF-SCO was carried out [32]. A combination of the Becke exchange plus Lee-Yang-Parr correlation functional (B3LYP) and all-electron core potentials has been used. Additionally, the double- $\zeta$ numerical polarization (DNP) basis set was adopted to account for the d-type into heavier atoms and p-type polarization into hydrogen atoms. This basis is similar to the 6-31G(d,p) Gaussian-type basis set. The partial charges were calculated from the Mulliken population analysis. Partial charges are reported in the Supporting Information (forcefield.txt). Let us mention that the charges were calculated by considering the spin state (quintet and singulet) of the MOF-SCO.

\section{Force Fields}

The MOF framework was kept rigid. The host-guest interactions were thus calculated by considering the electrostatic and the dispersive-repulsive potential. The dispersive-repulsive contribution was computed by using the Lennard-Jones (LJ) potential characterized by $\sigma$ (the finite distance at which the inter-particle potential is zero) and $\varepsilon$ (is the depth of the potential well). MOF-SCO material was modeled by combining the UFF and the DREIDING potential parameters considered for the inorganic and organic nodes respectively [33]. Benzaldehyde molecules were modeled by using the flexible OPLS/AMBER force field $[34,35]$. The LJ parameters are provided in the Supporting Information (forcefield.txt). The crossed LJ interactions between the benzaldehyde molecules and the MOF-SCO were calculated by means of the mixing Lorentz-Berthelot rules.

\section{Grand Canonical Monte Carlo (GCMC) Simulation}

Grand Canonical Monte Carlo (GCMC) simulations were considered to fill the porous material at 1 bar and $\mathrm{T}=298 \mathrm{~K}$ (saturation). These calculations were carried out using translational, rotational and insertion/deletion trial moves with frequencies of 0.2, 0.2 and 0.6, respectively. $1 \times 10^{9} \mathrm{GCMC}$ steps were necessary to obtain a convergence of the amount 
of adsorbed benzaldehyde. GCMC simulations were achieved by considering the fugacity of the benzaldehyde. In this work both HS and LS forms were considered as rigid. In both cases amount of adsorbed benzaldehyde was found to be equal to 8 molecules per unit cell i.e. 2 molecules per cage. MD simulation runs were conducted for $10 \mathrm{~ns}$ with a time step of 1 fs with 10 ns of equilibration. The equations of motion were integrated using the velocity Verlet scheme. The Ewald summation was used for calculating the electrostatic interactions. Eventually, the short-range interactions were truncated at $12 \AA$.

\section{Molecular Dynamics Simulations}

The simulation box consisted of 2 unit cells such as $L_{x}=30.533 \AA$ A,$L_{y}=30.533 \AA$ and $L_{z}=31.017 \AA$ for the LS form and $L_{x}=31.824 \AA, L_{y}=31.824 \AA$ and $L_{z}=31.022 \AA$ for the HS phase. $L_{x}, L_{y}$ and $L_{z}$ are the box lengths according to the $\mathrm{x}, \mathrm{y}$ and $\mathrm{z}$ directions respectively. Molecular Dynamics (MD) simulations were performed by using the $D L_{P O L Y}$ software [36] in the NVT ensemble where $\mathrm{N}$ is the number of molecules, $\mathrm{T}$ the temperature and $\mathrm{V}$ the volume. The Nose-Hoover thermostat was used with a relaxation time of 0.5 ps.

\section{Appendix B: Structure Determination}

CrysAlisPRO [23] software from Rigaku Oxford Diffraction was used for data processing: indexing, integration and reduction. During data processing a data scaling and empirical or multi-scan absorption corrections were applied. All structures were solved by direct methods using SHELXT, [37] and were refined by full-matrix least squares on $F^{2}$ using SHELXL, [38, 39] in $O L E X^{2}$ GUI [40]. All non-hydrogen atoms were refined anisotropically; H-atoms were constrained by geometry. When applicable, SIMU and ISOR restrains were applied for occupational disorder of NCS group. The guest molecules trapped in the voids of the host framework due to their strong disorder have been treated with PLATON SQUEEZE tool [41]. The unfitted electron density indicates the presence of max. $8 \mathrm{Ph}$ CHO molecules per one-unit cell. For the purpose of the article a choice of the average cell built on the Fe-host frame was made during the data treatment and the structure determination process. Symmetry has been assigned accordingly as $P 4 / n 21 / c 2 / c$ for $250 \mathrm{~K}$ data, and as $P \overline{4} 2_{1} c$ for $210 \mathrm{~K}, 195 \mathrm{~K}$ and $130 \mathrm{~K}$ data. CCDC-1970083 (250 K), CCDC- 
1970082 (210 K), CCDC-1970081 (195 K) and CCDC-1970080 (130 K) contain the crystal data collection and refinement parameters details for this paper, which can be obtained free of charge via www.ccdc.cam.ac.uk/conts/retrieving.html (or from the Cambridge Crystallographic Data Centre, 12 Union Road, Cambridge CB2 1EZ, UK; fax: (+44) 1223-336-033; or deposit@ccdc.ca.ac.uk).

\section{ACKNOWLEDGMENTS}

The authors thank Dmitry Chernyshov (SNBL beamline, ESRF) for his support during the synchrotron experiment. The authors acknowledge Jumana Shikh Almakara and Simon Le Mehauté for fruitful discussions. Thanks to the Spanish Ministerio de Economia y Competitividad (MINECO), FEDER (CTQ2016-78341-P), Unidad de Excelencia Mara de Maeztu (MDM-2015-0538), and the Generalitat Valenciana through PROMETEO/2016/147.

[1] S. Kaskel, ed., The Chemistry of Metal-Organic Frameworks. Synthesis, Characterization, and Applications, (two volumes) (WileyVCH, Weinheim, 2016).

[2] C. Zhao, X. Dai, T. Yao, W. Chen, X. Wang, J. Wang, J. Yang, S. Wei, Y. Wu, and Y. Li, Journal of the American Chemical Society 139, 8078 (2017).

[3] W. Jiang, Z. Liu, J.-W. Mei, B. Cui, and F. Liu, Nanoscale 11, 955 (2019).

[4] G. Férey and C. Serre, Chem. Soc. Rev. 38, 1380 (2009).

[5] A. Schneemann, V. Bon, I. Schwedler, I. Senkovska, S. Kaskel, and R. A. Fischer, Chem. Soc. Rev. 43, 6062 (2014).

[6] A. Hauser, Angewandte Chemie International Edition 52, 10419 (2013).

[7] A. Bousseksou, G. Molnár, L. Salmon, and W. Nicolazzi, Chem. Soc. Rev. 40, 3313 (2011).

[8] H. Watanabe, K. Tanaka, N. Bréfuel, H. Cailleau, J.-F. Létard, S. Ravy, P. Fertey, M. Nishino, S. Miyashita, and E. Collet, Phys. Rev. B 93, 014419 (2016).

[9] D. Chernyshov, M. Hostettler, K. W. Törnroos, and H.-B. Bürgi, Angewandte Chemie (International ed. in English) 42, 3825 (2003). 
[10] E. Collet, H. Watanabe, N. Bréfuel, L. Palatinus, L. Roudaut, L. Toupet, K. Tanaka, J.-P. Tuchagues, P. Fertey, S. Ravy, B. Toudic, and H. Cailleau, Phys. Rev. Lett. 109, 257206 (2012).

[11] S. Bonnet, M. A. Siegler, J. S. Costa, G. Molnár, A. Bousseksou, A. L. Spek, P. Gamez, and J. Reedijk, Chem. Commun. , 5619 (2008).

[12] J. E. Clements, J. R. Price, S. M. Neville, and C. J. Kepert, Angewandte Chemie International Edition 55, 15105 (2016).

[13] D. Chernyshov, H.-B. Bürgi, M. Hostettler, and K. W. Törnroos, Phys. Rev. B 70, 094116 (2004).

[14] M. Nishino, K. Boukheddaden, S. Miyashita, and F. Varret, Phys. Rev. B 68, 224402 (2003).

[15] N. Ortega-Villar, M. C. Muñoz, and J. A. Real, Magnetochemistry 2 (2016).

[16] T. Romero-Morcillo, N. DelaPinta, L. M. Callejo, L. Piñeiro López, M. C. Muoz, G. Madariaga, S. Ferrer, T. Breczewski, R. Cortés, and J. A. Real, Chemistry A European Journal 21, 12112 (2015).

[17] D. Zhang, E. Trzop, F. J. Valverde-Muñoz, L. Piñeiro López, M. C. Muñoz, E. Collet, and J. A. Real, Crystal Growth \& Design 17, 2736 (2017).

[18] J. A. Real, A. B. Gaspar, and M. C. Muñoz, Dalton Trans. , 2062 (2005).

[19] Z. Yan, Z.-P. Ni, F.-S. Guo, J.-Y. Li, Y.-C. Chen, J.-L. Liu, W.-Q. Lin, D. Aravena, E. Ruiz, and M.-L. Tong, Inorganic Chemistry 53, 201 (2014).

[20] P. D. Southon, L. Liu, E. A. Fellows, D. J. Price, G. J. Halder, K. W. Chapman, B. Moubaraki, K. S. Murray, J.-F. Létard, and C. J. Kepert, Journal of the American Chemical Society 131, 10998 (2009).

[21] E. Trzop, D. Zhang, L. Piñeiro Lopez, F. J. Valverde-Muñoz, M. C. Muñoz, L. Palatinus, L. Guérin, H. Cailleau, J. A. Real, and E. Collet, Angewandte Chemie International Edition 55, 8675 (2016).

[22] C. Mariette, E. Trzop, S. Zerdane, P. Fertey, D. Zhang, F. J. Valverde-Muñoz, J.-A. Real, and E. Collet, Acta Crystallographica Section B 73, 660 (2017).

[23] CrysalisPro v. 1.171.38.46, Rigaku Oxford Diffraction (2018).

[24] C. Adamo and V. Barone, The Journal of Chemical Physics 108, 664 (1998).

[25] M. J. Frisch, G. W. Trucks, H. B. Schlegel, G. E. Scuseria, M. A. Robb, J. R. Cheeseman, G. Scalmani, V. Barone, B. Mennucci, G. A. Petersson, and al., Gaussian, Inc., Pittsburgh, 
PA (2009).

[26] A. P. Scott and L. Radom, The Journal of Physical Chemistry 100, 16502 (1996).

[27] R. D. Johnson III, ed., NIST Computational Chemistry Comparison and Benchmark Database - NIST Standard Reference Database Number 101 (Release 20, August 2019).

[28] J. P. Merrick, D. Moran, and L. Radom, The Journal of Physical Chemistry A 111, 11683 (2007), pMID: 17948971.

[29] V. Barone, The Journal of Chemical Physics 122, 014108 (2005).

[30] V. Barone, M. Biczysko, J. Bloino, M. Borkowska-Panek, I. Carnimeo, and P. Panek, International Journal of Quantum Chemistry 112, 2185 (2012).

[31] J. J. Meinnel, A. Boudjada, A. Boucekkine, F. Boudjada, A. Moréac, and S. F. Parker, The Journal of Physical Chemistry A 112, 11124 (2008).

[32] Y. F. Chen, R. Babarao, S. I. Sandler, and J. W. Jiang, Langmuir 26, 8743 (2010).

[33] S. L. Mayo, B. D. Olafson, and W. A. Goddard, The Journal of Physical Chemistry 94, 8897 (1990).

[34] W. L. Jorgensen, D. S. Maxwell, and J. Tirado-Rives, Journal of the American Chemical Society 118, 11225 (1996).

[35] W. D. Cornell, P. Cieplak, C. I. Bayly, I. R. Gould, K. M. Merz, D. M. Ferguson, D. C. Spellmeyer, T. Fox, J. W. Caldwell, and P. A. Kollman, Journal of the American Chemical Society 117, 5179 (1995).

[36] I. T. Todorov, W. Smith, K. Trachenko, and M. T. Dove, J. Mater. Chem. 16, 1911 (2006).

[37] G. M. Sheldrick, Acta Crystallographica Section A 71, 3 (2015).

[38] G. M. Sheldrick, Acta Crystallographica Section A 64, 112 (2008).

[39] G. M. Sheldrick, Acta Crystallographica Section C 71, 3 (2015).

[40] O. V. Dolomanov, L. J. Bourhis, R. J. Gildea, J. A. K. Howard, and H. Puschmann, Journal of Applied Crystallography 42, 339 (2009).

[41] A. L. Spek, Acta Crystallographica Section C 71, 9 (2015). 